\title{
Lipopolysaccharide Reduces Incentive Motivation While Boosting Preference for High Reward in Mice
}

\author{
Elisabeth G Vichaya', Sarah C Hunt' and Robert Dantzer*,' \\ 'Division of Internal Medicine, Department of Symptom Research, The University of Texas MD Anderson Cancer Center, Houston, TX, USA
}

\begin{abstract}
Inflammation has been implicated in the development of various psychiatric disorders, including depression. However, the neurobehavioral mechanism involved in this relationship remains elusive. This gap in knowledge may best be filled by evaluating elementary neurobehavioral units affected by inflammation rather than behavioral changes in conventional animal tests of depression. To this end, the current study used a concurrent choice paradigm to evaluate inflammation-induced motivational changes. Male C57BL/6 mice $(n=27)$ were food restricted to between 85 and $90 \%$ of their free-feeding weight and were trained to perform a concurrent choice task where they nose-poked for grain rewards on a fixed ratio (FR) I schedule (low effort/low reward) and chocolate-flavored rewards on a FR-IO schedule (high effort/high reward). A counterbalanced-within subjects design was used. A single intraperitoneal injection of $0.33 \mathrm{mg} / \mathrm{kg}$ lipopolysaccharide (LPS) was used to induce peripheral inflammation. Twenty-four hours after LPS administration, mice showed a reduction in the total number of nose pokes. A proportionally greater reduction in nose pokes was observed for grain, resulting in an increase in percent chocolate pellets earned. These behavioral changes cannot be explained by reduced appetite as feeding before the test led to a similar increase in percent chocolate pellets earned but without any decrease in responding. These results indicate that inflammation modulates incentive motivation by affecting willingness to exert effort for reward and not by reducing sensitivity to reward.

Neuropsychopharmacology (2014) 39, 2884-2890; doi:I0.I038/npp.20 I4.I4I; published online 9 July 2014
\end{abstract}

\section{INTRODUCTION}

Inflammation has been implicated as a possible causal factor for the development of symptoms in a number of psychiatric disorders including schizophrenia, major depressive disorders, and bipolar disorders (Leboyer et al, 2012; Miller et al, 2009; Potvin et al, 2008). However, the neurobehavioral processes that are involved in this relationship are still elusive. The limiting factor is represented by the lack of information on the exact dimensions of mental disorders that are possibly influenced by inflammation. The research domain criteria (RDoCs) initiative that aims at describing mental disorders in terms of functions and neural circuit impairments (Cuthbert and Insel, 2010) is certainly better suited for addressing this knowledge gap than the usual description of effects of inflammation in terms of symptoms. Among the broad functional domains that constitute the rows of the RDoC matrix, the concept of reward-related behavior appears particularly valuable for addressing the nature of the behavioral impairment caused by inflammation. Inflammatory stimuli cause an apparent

* Correspondence: Dr R Dantzer, Division of Internal Medicine, Department of Symptom Research, The University of Texas MD Anderson Cancer Center, I 400 Pressler Road Unit 1450, Houston, TX 77030, USA, Tel: + I 713563 4793, Fax: + I 713745 3475,

E-mail: rdantzer@mdanderson.org

Received 16 December 2013; revised 14 May 2013; accepted 5 June 2014; accepted article preview online II June 2014 reduction in sensitivity to reward that can be evidenced by reduced preference for a sweetened solution (Frenois et al, 2007; Shen et al, 1999; Yirmiya, 1996) and decreased responding for a rewarding electrical stimulation of the lateral hypothalamus (Anisman et al, 1998; Borowski et al, 1998) in rodents injected with the cytokine inducer lipopolysaccharide (LPS) or recombinant proinflammatory cytokines. These effects of inflammation have been confirmed in humans. In response to a low dose of LPS, volunteers showed increases in self-reported and observerrated depressed mood over time and reduced ventral striatal activity to monetary reward cues (Eisenberger et al, 2010).

Although these findings appear to be in favor of a reduced sensitivity to reward, other interpretations are possible. A motivational account of the behavioral effects of endotoxin in rats was proposed by Miller (1964) fifty years ago. He observed that rats treated with endotoxin decreased lever pressing for food, water, and intracranial rewarding selfstimulation. However, the decrease in lever pressing was replaced by an increase when rats submitted to forced wheel running were trained to lever press to obtain periods of rest. These results led Miller to propose that endotoxin induces a motivational state of sickness that competes with other motivational states. This hypothesis was confirmed by Aubert et al (1997) using a situation of motivational competition in which sickness behavior was set to compete with maternal behavior. Lactating mice were injected with a sickness-inducing dose of LPS and the impact of this 
treatment on two measures of maternal behavior, pup retrieval and nest building, was tested at two different levels of motivation. As predicted by the motivational competition hypothesis, the effect of LPS depended on the priority of the different motivational states that were in competition. Since then, there have been many reports of the ability of LPSinduced sickness to interfere with motivated behavior. The proinflammatory cytokine interleukin-1 $\beta$ (IL-1 $\beta$ ) was found to decrease progressive ratio performance in mice, with a greater impact on high effort than low effort responding for milk (Larson et al, 2002). In addition, both IL-1 $\beta$ and LPS decreased sucrose intake but had no effect on sucroseinduced place preference (Larson, 2006), indicating that they do not interfere with the rewarding properties of sucrose. Taken together, these findings indicate that the motivational state of sickness induced by LPS decreases the incentive to engage in other types of motivated behavior that interfere with the priorities of a sick individual (Aubert, 1999).

Most behavioral tasks do not allow dissociating a decreased sensitivity to reward from a reduced motivation to obtain reward. However, the concurrent choice paradigm developed by Salamone allows for this dissociation (Cousins et al, 1994; Salamone et al, 1991). In this task, subjects have a choice between a high-effort/high-reward option (eg, lever pressing for a preferred reward) and a low-effort/ low-reward option (eg, consuming a freely available non-preferred reward). This strategy has been used to evaluate the mechanisms underlying incentive motivation, ie, the effort a subject is ready to engage in, in order to get a reward. Recently it has been shown that treatment with IL-1 $\beta$ causes an acute shift toward the low-effort/lowreward option in rats trained in the concurrent choice task (Nunes et al, 2013). Furthermore, a human analog of this task developed by Michael Treadway has demonstrated that depressed patients switch to a low-effort/low-reward strategy before non-depressed individuals (Treadway et al, 2012; Treadway et al, 2009).

The objective of this current study was to use the concurrent choice paradigm to inform the motivational changes induced by LPS at a time in which sickness behavior is no longer apparent yet depressive-like behavior persists. As expected, we observed that LPS-treated mice maintained their preference for the preferred reward yet worked less independent of response cost.

\section{MATERIALS AND METHODS}

\section{Experimental Subjects}

Male C57BL/6J mice were ordered from Jackson Laboratories at 8 weeks of age $(n=27)$. Mice were individually housed in a temperature- and humidity-controlled environment with a 12-h light:dark cycle (lights on at 0600 hours). After 1 week of environmental habituation and handling, mice were gradually food restricted to between 85 and $90 \%$ of their free-feeding weight and maintained this condition for the duration of the experiment.

\section{Drugs and Administration}

LPS (LPS; serotype 012:B8, Sigma-Aldrich, St Louis, MO) was dissolved in phosphate-buffered saline (PBS) at a concentration of $33 \mu \mathrm{g} / \mathrm{ml}$. Mice were treated with 0.33 $\mathrm{mg} / \mathrm{kg}$ LPS or PBS $24 \mathrm{~h}$ before testing. This dose of LPS was selected based on its ability to induce transient sickness behavior followed $24 \mathrm{~h}$ later by depressive-like behavior (Dantzer et al, 2008; Frenois et al, 2007).

\section{Concurrent Choice Training and Testing}

Concurrent choice training and testing were conducted in operant conditioning chambers equipped with two nosepoke response units and two reward units (Med Associates, St Albans, VT). Mice were trained and tested in the chambers for $20 \mathrm{~min}$ 4-5 times per week for 10 weeks. Chocolate-flavored Dustless Precision Pellets served as the preferred reward and grain-based Dustless Precision Pellets served as the non-preferred reward (20 mg, BioServ, Frenchtown, NJ). The relative rewarding value of these pellets was confirmed based on the preference of naïve mice for chocolate pellets when tested in a situation of free choice in the home cage. Training alternated between choice (both preferred and non-preferred rewards available) and no choice (only the preferred reward available). Mice were initially trained on a fixed ratio (FR)-1 schedule for both rewards. While the grain pellet delivery was maintained at a FR-1 schedule throughout, the response requirement for chocolate pellets was gradually increased to a FR-10 schedule. Training on the FR-1/FR-10 schedule continued until subjects exhibited stable responding. Nose poking and reward dispensing were monitored by sensors within the chambers and data were output by MedState Notation Software. The percent chocolate pellet preference was calculated using the following formula: (chocolate pellets)/ (chocolate + grain pellets $) \times 100$.

\section{Free-Feeding Task}

A free-feeding task was performed at baseline as well as following LPS or PBS concurrent choice testing. In this task, two dishes of pellets, one with 10 chocolate pellets and one with 10 grain pellets, were introduced to the home cage. The initial pellet selection, latency to consume the first pellet, and time to consume all chocolate pellets, with a maximal time of $5 \mathrm{~min}$, were recorded. If a mouse failed to consume all of the chocolate pellets within the time allotted, they were given a score of $300 \mathrm{~s}$.

\section{Locomotor Activity}

Locomotor activity was assessed $24 \mathrm{~h}$ after treatment with LPS or PBS immediately before concurrent choice testing. Mice were placed in a clean empty shoebox cage $(18.4 \times 29.2 \mathrm{~cm})$ and their activity was video recorded for $5 \mathrm{~min}$. The cage was divided into quadrants and the number of line crosses in $5 \mathrm{~min}$ was determined by a trained experimenter blind to experimental condition.

\section{Experimental Design}

A counterbalanced-within subject experimental design was used. Before any experimental manipulations, baseline concurrent choice performance was assessed and a freefeeding task was conducted. All mice were treated with both 
LPS and PBS, with half receiving the LPS treatment first and half receiving PBS first. The injections were separated by 1 week. Twenty-four hours after treatment, mice were tested for locomotor activity immediately followed by concurrent choice testing and then the free-feeding task. A pre-feeding experiment, with conditions counterbalanced over two sessions, was conducted to assess the impact of reduced food motivation on performance. In this experiment, subjects were pre-fed or not with standard rodent chow $1 \mathrm{~h}$ before concurrent choice testing. These experiments were conducted in two replications.

\section{Statistical Analysis}

Data were analyzed using SPSS (version 19, Chicago, IL). Descriptive statistics are presented as mean \pm SEM. Twoway ANOVAs with a within-subjects treatment condition factor (vehicle $v s$ LPS) and a between-subjects block factor (replication 1 vs replication 2) were used to analyze concurrent choice and free-feeding performance $24 \mathrm{~h}$ following LPS or saline and following pre-feeding. Trend analyses of performance under the influence of LPS, controlling for saline performance, across time (ie, baseline, $24 \mathrm{~h}$ post, and $48 \mathrm{~h}$ post) were conducted to assess recovery from LPS.

\section{RESULTS}

\section{Concurrent Choice and Free-Feeding Behavior following} LPS Treatment

LPS treatment decreased the total number of pellets earned, $F(1,25)=84.6, p<0.001$, from an average of 48 pellets to 28 pellets in a 20 -min session. There was an approximately $43 \%$ reduction in nose pokes for grain, $F(1,25)=54.4, p<0.001$ (Figure 1a), and a $24 \%$ reduction in nose pokes for chocolate, $F(1,25)=6.43, p<0.05$ (Figure $1 b$ ), in response to LPS. Furthermore, there was a reduction in the total number of nose pokes, $F(1,25)=11.5, p<0.005$ (Figure 1c). Given the proportionally greater reduction in nose pokes for grain than for chocolate, there was an increase in percent chocolate pellets earned, $F(1,25)=11.8, p<0.005$ (Figure 1d). While there was no effect of replication on the number of nose pokes, the mice from replication 2 consumed fewer total pellets than the mice from replication $1, F(1,25)=7.93, p<0.01$. There were no significant interactions between any of the above variables and replication.

A trend analysis of the time course of behavioral changes measured immediately before injection (baseline), 24 and $48 \mathrm{~h}$ after LPS in relation to saline revealed a significant quadratic relationship for the total number of nose pokes $(F(1,24)=5.35, p<0.05$; Figure $2 \mathrm{a})$ and for percent chocolate pellets earned $(F(1,24)=12.0, p<0.01$; Figure $2 \mathrm{~b})$. This suggests that by $48 \mathrm{~h}$, mice displayed significant recovery. There was a trend toward a quadratic relationship between the number of nose pokes for grain pellets $(p<0.10)$; however, a quadratic relationship was not observed for nose pokes for chocolate pellets.

Following the 24-h post-treatment concurrent choice testing, mice were given free access to 10 chocolate and 10 grain pellets in their home cage. Neither group showed a significant change in preference for chocolate pellets in this test as defined by latency to begin eating chocolate pellets,
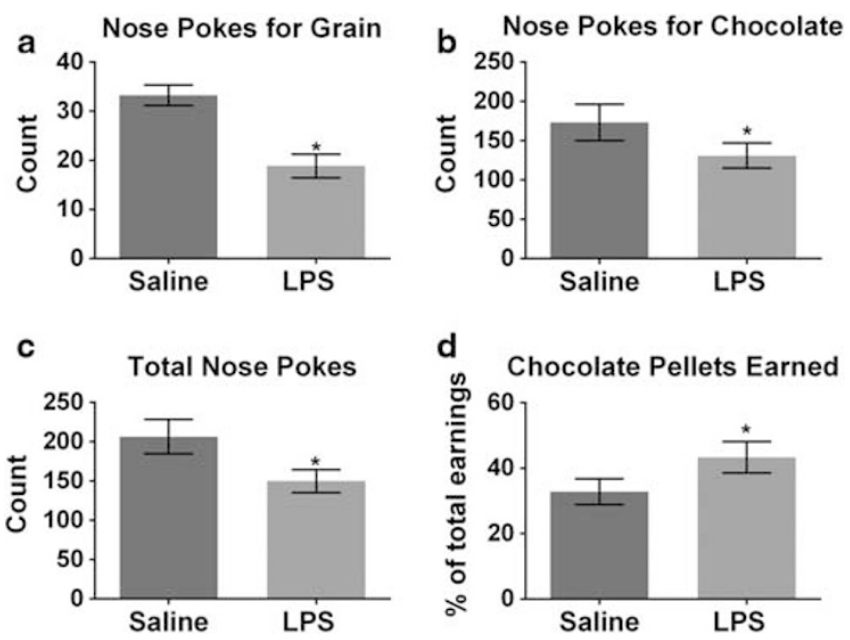

Figure I LPS treatment reduces total effort without loss of reward sensitivity. Concurrent choice performance was assessed $24 \mathrm{~h}$ following LPS or saline treatment. LPS decreased the number of nose pokes for grain (a) and chocolate (b), and the total nose poke number (c) while increasing the percent chocolate pellets earned (d). ${ }^{*} p<0.05$.
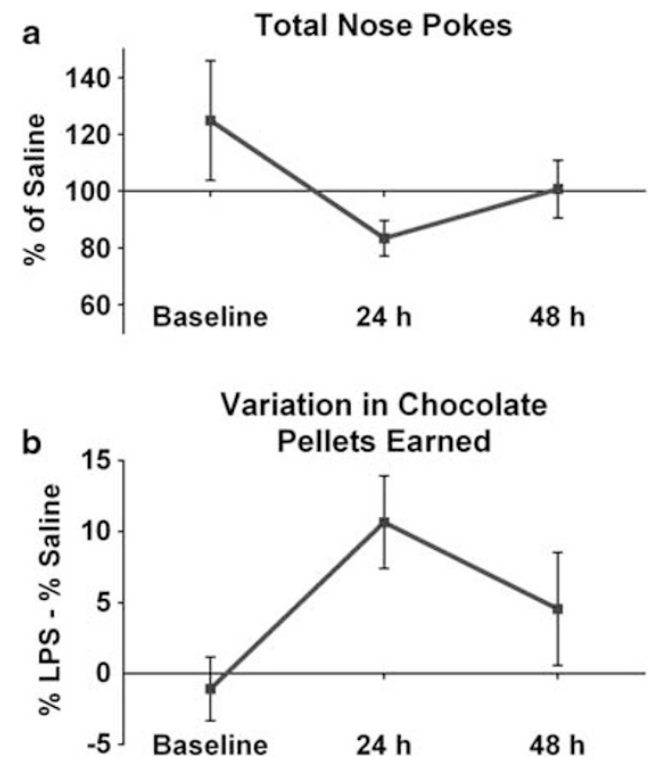

Figure 2 Time course of the effect of LPS in the concurrent choice task. A significant recovery of nose-poke performance, expressed as a percent of saline performance, (a) and change in percent chocolate rewards earned between LPS and saline conditions (b) was observed by $48 \mathrm{~h}$ post LPS treatment as indicated by significant across time quadratic relationships $(p<0.05)$.

latency to consume chocolate pellets, and percent chocolate pellets consumed (Figure 3a-c). However, the number of pellets consumed tended to decrease following LPS treatment $(p<0.10)$.

\section{General Effects of LPS}

At $24 \mathrm{~h}$ after LPS treatment, there was no evidence of significant body weight loss. However, LPS treatment did result in less horizontal activity in a new cage compared 

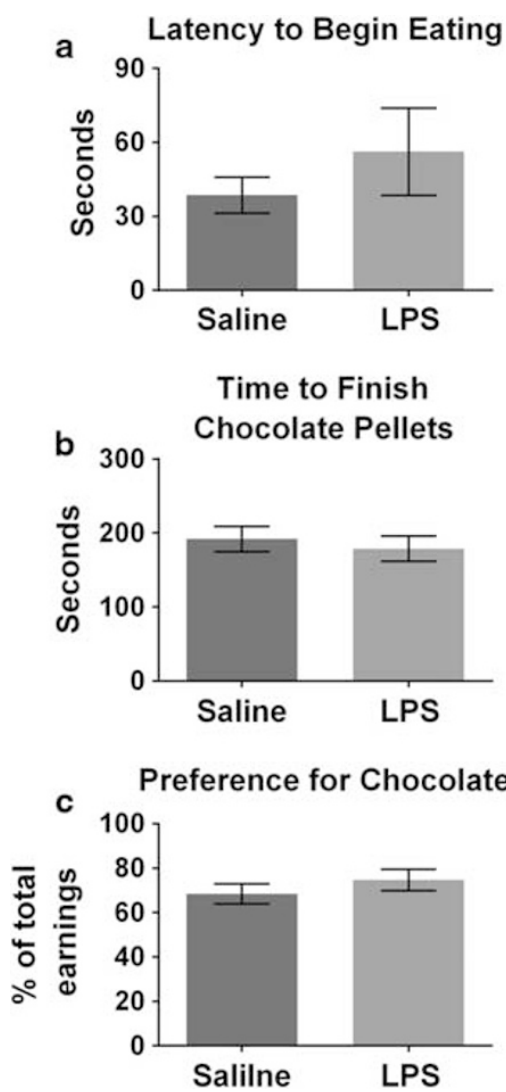

Figure 3 LPS does not alter free-feeding behavior. Two dishes of pellets were placed in the home cage, one containing 10 chocolate pellets and one containing 10 grain-based pellets. The latency to begin eating the pellets (a), the time required to consume all of the chocolate pellets (b), and percent chocolate pellets consumed (c) were not significantly affected by LPS treatment.

with vehicle-treated mice $(61.3 \pm 3.29$ vs $90.2 \pm 4.13$ line crossings in $5 \mathrm{~min}), F(1,25)=27.3, p<0.001$.

\section{Concurrent Choice Responding Following Pre-Feeding}

Pre-feeding for $1 \mathrm{~h}$ before concurrent choice testing was conducted to evaluate the impact of a motivational shift on concurrent choice performance. Pre-feeding resulted in a reduction in the number of nose pokes for grain $(F(1,25)=56.1, p<0.001$, Figure $4 \mathrm{a})$. The number of nose pokes for chocolate was not significantly changed (Figure 4b) nor was the total number of nose pokes (Figure 4c). The subtle shifting away from nose pokes for grain toward nose pokes for chocolate resulted in an increase in percent of chocolate pellets earned, $F(1,25)=10.4, p<0.01$ (Figure $4 d$ ). No significant interactions were observed between any of the tested variables and experimental replication. However, mice in round 2 consumed fewer pellets and nose-poked less for grain than mice from replication 1 .

\section{DISCUSSION}

To inform the motivational changes induced by LPS, we tested mice in a concurrent choice paradigm with lowcost (FR-1)/low-reward (grain-based pellet) and high-cost
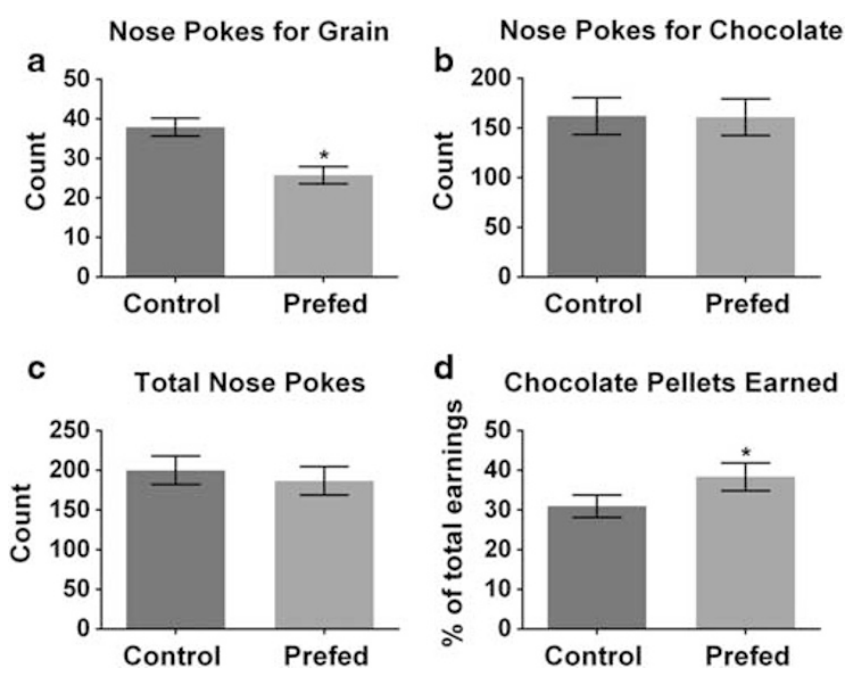

Figure 4 Effect of pre-feeding on performance in the concurrent choice task. Concurrent choice performance was tested following pre-feeding and control conditions. Pre-feeding decreased the number of nose pokes for grain (a), but did not have a significant effect on nose pokes for chocolate (b) or total nose poke number (c). Pre-feeding also increased the percent chocolate pellets earned (d). ${ }^{*} p<0.05$.

(FR-10)/high-reward (chocolate-flavored pellet) options. LPS treatment resulted in a reduction in total effort for rewards but did not reduce sensitivity to the reward.

Significant individual variability in choice strategy was observed in the concurrent choice paradigm. These differences cannot be explained by differences in the hedonic value of the reinforcer, as chocolate pellets were consistently preferred to grain pellets when given free access to both types in their home cage. Our study is not the first to observe high variability in operant performance. For example, Randall et al (2012) report performance that varies from fewer than 100 to over 1000 lever presses in a testing session. They suggest this variability may relate to neurochemical differences. Further, the drug self-administration literature has also described significant variability in performance and has related this variability to addiction potential (Deminiere et al, 1989; Mitchell et al, 2014). While the characterization of this variability is outside the scope of this manuscript, it is within the range of what has been previously reported.

LPS-treated mice showed a reduction in motor activity when compared with the vehicle-treated controls. Generally, this effect would be interpreted as a reduction in general activity associated with lingering sickness behavior. However, the large size of this effect is inconsistent with what we observed in our previous studies on LPS-induced sickness and depression-like behavior. Decreases in activity, when they occur, are usually small and the level of activity in control mice is in the order of 50 rather than 80 line crossing in $5 \mathrm{~min}$ (O'Connor et al, 2009a, b; Walker et al, 2013). Given that mice were tested for locomotor activity immediately before concurrent choice testing, the elevation in activity measured in the present study may be anticipatory activity, as observed before feeding in mice submitted to restricted-feeding schedules (AngelesCastellanos et al, 2008; Wakamatsu et al, 2001). Therefore, 
the activity increase observed in vehicle-treated conditions is likely related to food motivation, and the suppression observed following LPS treatment is probably an indirect measure of reduced incentive motivation. Alternatively, this reduction in activity could be related to an LPS-induced impairment in the expression of gene clocks that are entrained by food availability (Angeles-Castellanos et al, 2008; Yamamura et al, 2010).

The decreased effort measured by the total number of nose pokes in response to LPS could have been caused by lingering effects of LPS on appetite. LPS is well known to decrease food appetite via both peripheral effects, including inhibition of gastric emptying and acceleration of intestinal transit, and central effects (Asarian and Langhans, 2010; Langhans, 1996). However, most of these effects are observed rapidly after administration of LPS, whereas mice were tested $24 \mathrm{~h}$ after LPS in the present experiment, a time at which their food intake would be expected to have recovered (Dantzer et al, 2008; Frenois et al, 2007; O'Connor et al, 2009c). The mice in the current study still engaged in consummatory behavior in the free-feeding task immediately following the concurrent choice task, indicating they were not fully satiated at the end of the task. When the mice were pre-fed to test the effect of reduced appetite on performance, a boost in preference for chocolate pellets was observed but a decrease in the total effort for earning food pellets in the concurrent performance task was not observed. This last effect was in contrast to the effect of LPS. Taken together, these data suggest that a decrease in appetite is insufficient to explain the motivational changes observed in response to LPS treatment.

Decreased performance in a food-motivated task can be influenced by either a change in an individual's sensitivity to reward (ie, 'liking') or a deficit in the initiation or maintenance of goal-directed behavior (ie, 'wanting'). The terms liking and wanting are used here as defined by Berridge (Berridge, 1996, 2009). If LPS had affected motivated behavior by reducing liking, a decrease in responding for chocolate relative to responding for grain should have been observed. Furthermore, a decreased preference for chocolate pellets in the free-feeding task should have been apparent. However, this was not the pattern of results we observed. Further evidence indicating that LPS does not induce changes in liking comes from two studies that investigated the effects of LPS on hedonic responses of rats to the taste of sweetness (Aubert and Dantzer, 2005; Cross-Mellor et al, 2004). In both cases, the taste reactivity to orally infused sweetness was unchanged.

The possibility that LPS reduces wanting or incentive motivation rather than liking is apparent from prior work demonstrating that the inflammatory cytokine IL-1 $\beta$, which mediates the behavioral effects of LPS (Dantzer, 2001), is capable of reducing operant responding for food, especially in tasks requiring a large effort (Larson et al, 2002; Merali et al, 2003; Nunes et al, 2013). In a direct test of the incentive motivation hypothesis, rats treated with IL-1 $\beta$ and tested at the peak of their sickness response decreased their operant responding for preferred food pellets but increased their consumption of freely available regular rodent chow (Nunes et al, 2013). These results were interpreted as indicating that IL-1 $\beta$-treated rats have an unchanged appetite but reallocate their behavior away from high effort responding for earning highly valued food to a less-effortful responding for obtaining less-valued food. However, the problem with the paradigm used to demonstrate this effect is that it compares operant responding for food pellets, which is the equivalent of appetitive behavior, with intake of free food, which is the equivalent of consummatory behavior. There is already evidence that immune stimuli preferentially affect appetitive over consummatory behavior (Cross-Mellor et al, 1999, 2000). To avoid this bias, we modified the paradigm to allow us to directly compare high effort/high reward to low effort/low reward using the same type of effort requirement, nose poking. With this task we observed that LPS did not cause a shift from high effort/high reward to low effort/low reward. Instead, LPS redirected the diminished effort that was still emitted to the reward that had the higher value. This is in agreement with the observation that the value of a reward is not merely a function of its intrinsic features but also of the effort that is necessary to obtain it (Johnson and Gallagher, 2011). A previous experience of a greater effort for a given food boosts the affective taste properties of that food as demonstrated in mice submitted to various relationships between effort and reinforcer value (Johnson and Gallagher, 2011). The altered motivational state induced by inflammation may induce a fatigue-like state that has been noted to result in a reprioritization of effort toward the more valued response (Taylor and Kielhofner, 2003). However, it is also possible that this behavioral change may parallel inefficiencies in cost/benefit decision making that have been observed in patients with major depressive disorder (Treadway et al, 2012).

In the present study, we did not address the neurobiological mechanisms that mediate the effects of LPS. It is already known that decreased incentive motivation is largely mediated by changes in dopamine within the nucleus accumbens (Farrar et al, 2010; Salamone et al, 2007). Inflammation may modulate dopamine neurotransmission through a variety of mechanisms including direct effects on dopamine reuptake or the function and/or expression of the vesicular monoamine transporter within dopaminergic neurons (Capuron et al, 2012; Felger and Miller, 2012; Kamata et al, 2000; Kitagami et al, 2003; Shuto et al, 1997). Furthermore, LPS-induced cytokines may influence dopamine neurotransmission via the formation of neurotoxic kynurenine metabolites produced downstream of the activation of the tryptophan-degrading enzyme indoleamine 2,3-dioxygenase (IDO) (Dantzer et al, 2008; Felger and Miller, 2012).

In conclusion, the present findings show that LPS reduces incentive motivation and redirects the reduced effort toward highly preferred rewards. As this effect is apparent at a time at which LPS induces depression-like behavior, the next question of importance is the relationship of this effect with inflammation-induced depression and its possible dependence on IDO activation. In terms of depression, it should be clear now that LPS does not induce a general state of anhedonia but reduces incentive motivation. This effect could be related to the anergia component of fatigue and depression, as already proposed (Nunes et al, 2013). In terms of mechanisms, there is much that still needs to be done using pharmacological and genetic tools to determine whether the reduced incentive motivation and the boosting 
of preference for high rewards are two facets of the same phenomenon or two independent processes.

\section{FUNDING AND DISCLOSURE}

This work was supported by the University of Texas MD Anderson Cancer Center and grants from the National Institute of Neurological Diseases and Stroke of the National Institutes of Health (Grants R01 NS073939; R01 NS074999). The content is solely the responsibility of the authors and does not necessarily represent the official view of the National Institutes of Health. RD consulted for Ironwood Pharma (Cambridge, MA).

\section{REFERENCES}

Angeles-Castellanos M, Salgado-Delgado R, Rodriguez K, Buijs RM, Escobar C (2008). Expectancy for food or expectancy for chocolate reveals timing systems for metabolism and reward. Neuroscience 155: 297-307.

Anisman H, Kokkinidis L, Borowski T, Merali Z (1998). Differential effects of interleukin (IL)-1 $\beta$, IL-2 and IL-6 on responding for rewarding lateral hypothalamic stimulation. Brain Res 779: 177-187.

Asarian L, Langhans W (2010). A new look on brain mechanisms of acute illness anorexia. Physiol Behav 100: 464-471.

Aubert A (1999). Sickness and behaviour in animals: a motivational perspective. Neurosci Biobehav Rev 23: 1029-1036.

Aubert A, Dantzer R (2005). The taste of sickness: lipopolysaccharide-induced finickiness in rats. Physiol Behav 84: 437-444.

Aubert A, Goodall G, Dantzer R, Gheusi G (1997). Differential effects of lipopolysaccharide on pup retrieving and nest building in lactating mice. Brain Behav Immun 11: 107-118.

Berridge KC (1996). Food reward: brain substrates of wanting and liking. Neurosci Biobehav Rev 20: 1-25.

Berridge KC (2009). 'Liking'and 'wanting'food rewards: brain substrates and roles in eating disorders. Physiol Behav 97: 537-550.

Borowski T, Kokkinidis L, Merali Z, Anisman H (1998). Lipopolysaccharide, central in vivo biogenic amine variations, and anhedonia. Neuroreport 9: 3797-3802.

Capuron L, Pagnoni G, Drake DF, Woolwine BJ, Spivey JR, Crowe $\mathrm{RJ}$ et al (2012). Dopaminergic mechanisms of reduced basal ganglia responses to hedonic reward during interferon alfa administrationreduced basal ganglia responses to hedonic reward. Arch Gen Psychiatry 69: 1044-1053.

Cousins MS, Wei W, Salamone JD (1994). Pharmacological characterization of performance on a concurrent lever pressing/feeding choice procedure: effects of dopamine antagonist, cholinomimetic, sedative and stimulant drugs. Psychopharmacology (Berl) 116: 529-537.

Cross-Mellor SK, Kavaliers M, Ossenkopp K-P (2004). Comparing immune activation (lipopolysaccharide) and toxin (lithium chloride)-induced gustatory conditioning: lipopolysaccharide produces conditioned taste avoidance but not aversion. Behavi Brain Res 148: 11-19.

Cross-Mellor SK, Kent WD, Kavaliers M, Ossenkopp K-P (2000). Examining the effects of lipopolysaccharide and cholecystokinin on water ingestion: comparing intake and palatability. Brain Res 861: 220-232.

Cross-Mellor SK, Kent WD, Ossenkopp K-P, Kavaliers M (1999). Differential effects of lipopolysaccharide and cholecystokinin on sucrose intake and palatability. Am J Physiol 277: R705-R715.

Cuthbert BN, Insel TR (2010). Toward new approaches to psychotic disorders: the NIMH research domain criteria project. Schizophr Bull 36: 1061-1062.
Dantzer R (2001). Cytokine-induced sickness behavior: where do we stand? Brain Behav Immun 15: 7-24.

Dantzer R, O'Connor JC, Freund GG, Johnson RW, Kelley KW (2008). From inflammation to sickness and depression: when the immune system subjugates the brain. Nat Rev Neurosci 9: 46-56.

Deminiere JM, Piazza PV, Le Moal M, Simon H (1989). Experimental approach to individual vulnerability to psychostimulant addiction. Neurosci Biobehav Rev 13: 141-147.

Eisenberger NI, Berkman ET, Inagaki TK, Rameson LT, Mashal NM, Irwin MR (2010). Inflammation-induced anhedonia: endotoxin reduces ventral striatum responses to reward. Biol Psychiatry 68: 748-754.

Farrar A, Segovia K, Randall P, Nunes EJ, Collins LE, Stopper CM et al (2010). Nucleus accumbens and effort-related functions: behavioral and neural markers of the interactions between adenosine $\mathrm{A}<$ sub $>2 \mathrm{~A}</$ sub $>$ and dopamine $\mathrm{D}<$ sub $>2<1$ sub $>$ receptors. Neuroscience 166: 1056-1067.

Felger JC, Miller AH (2012). Cytokine effects on the basal ganglia and dopamine function: the subcortical source of inflammatory malaise. Front Neuroendocrinol 33: 315-327.

Frenois F, Moreau M, O'Connor J, Lawson M, Micon C, Lestage J et al (2007). Lipopolysaccharide induces delayed FosB/DeltaFosB immunostaining within the mouse extended amygdala, hippocampus and hypothalamus, that parallel the expression of depressive-like behavior. Psychoneuroendocrinology 32: 516-531.

Johnson AW, Gallagher M (2011). Greater effort boosts the affective taste properties of food. Proc R Soc B 278: 1450-1456.

Kamata M, Higuchi H, Yoshimoto M, Yoshida K, Shimizu T (2000). Effect of single intracerebroventricular injection of $\alpha$-interferon on monoamine concentrations in the rat brain. Eur Neuropsychopharmacol 10: 129-132.

Kitagami T, Yamada K, Miura H, Hashimoto R, Nabeshima T, Ohta T (2003). Mechanism of systemically injected interferonalpha impeding monoamine biosynthesis in rats: role of nitric oxide as a signal crossing the blood-brain barrier. Brain Res 978: 104-114.

Langhans W (1996). Bacterial products and the control of ingestive behavior: clinical implications. Nutrition 12: 303-315.

Larson SJ (2006). Lipopolysaccharide and interleukin-1 $\beta$ decrease sucrose intake but do not affect expression of place preference in rats. Pharmacol Biochem Behav 84: 429-435.

Larson SJ, Romanoff RL, Dunn AJ, Glowa JR (2002). Effects of interleukin- $1 \beta$ on food-maintained behavior in the mouse. Brain Behav Immun 16: 398-410.

Leboyer M, Soreca I, Scott J, Frye M, Henry C, Tamouza R et al (2012). Can bipolar disorder be viewed as a multi-system inflammatory disease? J Affect Disord 141: 1-10.

Merali Z, Brennan K, Brau P, Anisman H (2003). Dissociating anorexia and anhedonia elicited by interleukin-1 $\beta$ : antidepressant and gender effects on responding for "free chow "and earned" sucrose intake. Psychopharmacology (Berl) 165: 413-418.

Miller AH, Maletic V, Raison CL (2009). Inflammation and its discontents: the role of cytokines in the pathophysiology of major depression. Biol Psychiatry 65: 732-741.

Miller NE (1964). Some psychophysiological studies of motivation and of the behavioural effects of illness. Bull Brit Psychol Soc 17: 1271-1278.

Mitchell MR, Weiss VG, Beas BS, Morgan D, Bizon JL, Setlow B (2014). Adolescent risk taking, cocaine self-administration, and striatal dopamine signaling. Neuropsychopharmacology 39: 955-962.

Nunes EJ, Randall PA, Estrada A, Epling B, Hart EE, Lee CA et al (2013). Effort-related motivational effects of the pro-inflammatory cytokine interleukin 1-beta: studies with the concurrent fixed ratio 5/chow feeding choice task. Psychopharmacology (Berl) 231: 727-736. 
O’Connor JC, André C, Wang Y, Lawson MA, Szegedi SS, Lestage J et al (2009a). Interferon- $\gamma$ and tumor necrosis factor- $\alpha$ mediate the upregulation of indoleamine 2, 3-dioxygenase and the induction of depressive-like behavior in mice in response to bacillus Calmette-Guérin. J Neurosci 29: 4200-4209.

O'Connor JC, Lawson MA, André C, Briley EM, Szegedi SS, Lestage J et al (2009b). Induction of IDO by bacille CalmetteGuerin is responsible for development of murine depressive-like behavior. J Immunol 182: 3202-3212.

O'Connor JC, Lawson MA, Andre C, Moreau M, Lestage J, Castanon $\mathrm{N}$ et al (2009c). Lipopolysaccharide-induced depressive-like behavior is mediated by indoleamine 2,3-dioxygenase activation in mice. Mol Psychiatry 14: 511-522.

Potvin S, Stip E, Sepehry AA, Gendron A, Bah R, Kouassi E (2008). Inflammatory cytokine alterations in schizophrenia: a systematic quantitative review. Biol Psychiatry 63: 801-808.

Randall PA, Pardo M, Nunes EJ, Lopez Cruz L, Vemuri VK, Makriyannis A et al (2012). Dopaminergic modulation of effortrelated choice behavior as assessed by a progressive ratio chow feeding choice task: pharmacological studies and the role of individual differences. PloS one 7: e47934.

Salamone JD, Correa M, Farrar A, Mingote SM (2007). Effortrelated functions of nucleus accumbens dopamine and associated forebrain circuits. Psychopharmacology (Berl) 191: 461-482.

Salamone JD, Steinpreis RE, McCullough LD, Smith P, Grebel D, Mahan K (1991). Haloperidol and nucleus accumbens dopamine depletion suppress lever pressing for food but increase free food consumption in a novel food choice procedure. Psychopharmacology (Berl) 104: 515-521.

Shen Y, Connor TJ, Nolan Y, Kelly JP, Leonard BE (1999). Differential effect of chronic antidepressant treatments on lipopolysaccharide-induced depressive-like behavioural symptoms in the rat. Life Sci 65: 1773-1786.

Shuto H, Kataoka Y, Horikawa T, Fujihara N, Oishi R (1997). Repeated interferon- $\alpha$ administration inhibits dopaminergic neural activity in the mouse brain. Brain Res 747: 348-351.

Taylor RR, Kielhofner GW (2003). An occupational therapy approach to persons with chronic fatigue syndrome: part two, assessment and intervention. Occup Ther Health Care 17: 63-87.

Treadway MT, Bossaller NA, Shelton RC, Zald DH (2012). Effortbased decision-making in major depressive disorder: a translational model of motivational anhedonia. J Abnorm Psychol 121: 553-558.

Treadway MT, Buckholtz JW, Schwartzman AN, Lambert WE, Zald DH (2009). Worth the 'EEfRT'? the effort expenditure for rewards task as an objective measure of motivation and anhedonia. PloS one 4: e6598.

Wakamatsu H, Yoshinobu Y, Aida R, Moriya T, Akiyama M, Shibata S (2001). Restricted-feeding-induced anticipatory activity rhythm is associated with a phase-shift of the expression of mPer1 and mPer2 mRNA in the cerebral cortex and hippocampus but not in the suprachiasmatic nucleus of mice. Eur $J$ Neurosci 13: 1190-1196.

Walker AK, Budac DP, Bisulco S, Lee AW, Smith RA, Beenders B et al (2013). NMDA receptor blockade by ketamine abrogates lipopolysaccharide-induced depressive-like behavior in C57BL/ 6J Mice. Neuropsychopharmacology 38: 1609-1616.

Yamamura Y, Yano I, Kudo T, Shibata S (2010). Time-dependent inhibitory effect of lipopolysaccharide injection on Per1 and Per2 gene expression in the mouse heart and liver. Chronobiol Int 27: 213-232.

Yirmiya R (1996). Endotoxin produces a depressive-like episode in rats. Brain Res 711: 163-174. 